\title{
BMC Veterinary Research reviewer acknowledgement 2014
}

Hayley Henderson

\author{
Leticia Abecia \\ Spain \\ Gerardo Acosta \\ Chile \\ Pier Luigi Acutis \\ Italy \\ Alexandra Adams \\ UK \\ Hippolyte Affognon \\ Kenya \\ Claudio Afonso \\ USA \\ Jose Aguilera \\ Spain \\ Hiroyuki Akagi \\ Japan \\ Pablo Alarcon \\ UK \\ Samer Alasaad \\ Spain
}

Séverine Albouy-Boyer

Canada

Noelia Aldai

Spain

Karin Allenspach

UK

Julio Alvarez

Spain

Gema Alvarez-García

Spain

\author{
Carlos Ambrosio \\ Brazil \\ Burim Ametaj \\ Canada \\ Hermann Ammer \\ Germany \\ Janetrix Hellen Amuguni \\ USA \\ Arturo Anadon \\ Spain \\ Kevin Anderson \\ USA \\ Magnus Carl Andersson \\ Finland \\ Gunther Antonissen \\ Belgium \\ Luis Antunes \\ Portugal \\ Haseeb Anwar \\ Pakistan \\ Michal Arabski \\ Poland \\ Virginia Aragon \\ Spain \\ Toshiro Arai \\ Japan \\ Debra Archer \\ UK \\ David Argyle \\ UK
}

Itamar Aroch

Israel

Margarete Arras

Switzerland

Karin Artursson

Sweden

Chris Ashwell

USA

Spiridoula Athanasiadou

UK

Graeme Attwood

New Zealand

Joerg Auer

Switzerland

Gaël Auray

Switzerland

Mian Muhammad Awais

Pakistan

Chrisostom Ayebazibwe

Uganda

Ignacio Badiola

Spain

Kris Baert

Belgium

Wolfgang Baeumer

USA

Emilia Bagnicka

Poland

Tamás Bakonyi

Hungary

Correspondence: Hayley.henderson@biomedcentral.com

BioMed Central, Floor 6, 236 Gray's Inn Road, London WC1X 8HB, UK 
Vinayagamurthy Balamurugan

India

Udeni Balasuriya

USA

Alfonso Baldi
Italy
Cynthia Baldwin
USA

Anne Balkema-Buschmann

Germany

Hywel Ball

UK

Ray Ball

USA

Jacek Bania

Poland

Claudio Barbeito

Argentina

Lisa Barco

Italy

Alistair Barr

UK

Maria Barrandeguy

Argentina

Animesh Barua

USA

Giuseppe Baselli

Italy

Anna Bassols

Spain

Peter Bates

UK

John Bauer

USA

Maximilian Baumann

Germany

Gregoy Bedecarrats

Canada

Aynun Begum

USA

Elsa Beltran

UK

Livia Benato

UK
David Bennett

UK

Olaf Berke

Canada

Dirk Berkvens

Belgium

Emma Bermingham

New Zealand

Paul Bessell

UK

V Bhanuprakash

India

Tomasz Bielecki

Poland

Barbara Biller

USA

Sarah Billeter

USA

John Bingham

Australia

Helen Birch

UK

Karyn Bischoff

USA

Francois Blachier

France

Pat Blackall

Australia

Barbara Blacklaws

UK

Damer Blake

UK

Sandra Blome

Germany

Gerd Bobe

USA

Barbara Bockstahler

Austria

Michael Böer

Germany

Patrick Boerlin

Canada

Josef Böhm

Austria
Anders Miki Bojesen

Denmark

J. Elizabeth Bolhuis

Netherlands

Diego Bonatto

Brazil

Alexandro Bonifaz

Mexico

Stephanie Booth

Canada

Manuel Vitor Borca

USA

Nicole Borel

Switzerland

Susanne Boroffka

Netherlands

Luca Borrelli

Italy

Uberto Bortolotti

Italy

Tim Bosmans

Belgium

Gianluca Bossi

Italy

Adrian Boswood

UK

Filip Boyen

Belgium

Barry Bradford

USA

Daniel Bradway

USA

Gudrun Brandes

Germany

Marina Brash

Canada

Jackie Brearley

UK

Andrew Breed

UK

Walter Brehm

Germany

Klaus Brehm

Germany 
Julia Breuer

Germany

Gert Breur

USA

Robert Briggs

USA

Victor Brugman

UK

Sebastien Buczinski

Canada

Hubert Buczkowski

UK

Steven Budsberg

USA

Rikke Buhl

Denmark

Franciszek Burdan

Poland

Laura Burgin

UK

Eric Burrough

USA

Neil Burton

UK

Patrick Butaye

Belgium

L Catalina Cabrera

USA

Silvia Cabrera

Spain

Petra Cagnardi

Italy

Jianping Cai

China

Hugh Cai

Canada

Luigi Calamari

Italy

Paolo Calistri

Italy

Luis Calvinho

Argentina

Begoña Calvo

Spain
Zongxi Cao

China

Vittorio Capello

Italy

Tereza Cardoso

Brazil

Jorge U. Carmona

Colombia

Noèlia Carrasco

Spain

Angel L. Carrascosa

Spain

Jose M Carrillo Poveda

Spain

Jorge Castro

Spain

Elena Catelli

Italy

Lissandra Cavalli

Brazil

Vladimir Celer

Czech Republic

Jose Ceron

Spain

Giuliano Cerulli

Italy

Chanhee Chae

Korea, South

Chia-Yi Chang

Taiwan

Shih-Chieh Chang

Taiwan

Guillaume Chanoit

UK

Aspinas Chapwanya

Ireland

Carole Charlier

Belgium

Johannes Charlier

Belgium

Hso-Chi Chaung

Taiwan

Huanchun Chen

China
Hong Chen

China

Yaosheng Chen

China

Anik Chevrier

Canada

Adeline Chia

Malaysia

Mario Chiari

Italy

Ming-Tang Chiou

Taiwan

Tomasz Chmielewski

Poland

Bhudipa Choudhury

UK

Francesco Cian

UK

Louise Clark

UK

Sarah Cleaveland

UK

Archie Clements

Australia

Alison Clode

USA

Joan Coates

USA

Hans Coetzee

USA

Gerald Coles

UK

Philippe Colson

France

Gavin Conant

USA

Anthony Confer

USA

Franz Josef Conraths Germany

Ronald Corbee

Netherlands

Guillaume Counotte

Netherlands 
Wendel Coura-Vital

Brazil

Christopher Cowled

Australia

Vasile Cozma

Romania

Carolyn Cray

USA

Pascale Crepieux

France

Juan Cuervo-Arango

Spain

William Culp

USA

Roman Dabrowski

Poland

Trachsel Dagmar

France

Benjamin Dainat

Switzerland

Stefano D'Amelio

Italy

Sven Dänicke

Germany

Andrew Davidson

UK

Peter Davies

USA

Andrew Davison

UK

Lucy Davison

UK

Astrid De Greeff

Netherlands

Ario De Marco

Slovenia

Stefaan De Smet

Belgium

Stephanie De Vleeschauwer

Belgium

Clazien J. De Vos

Netherlands

Theo De Waal

Ireland
Nicola Decaro

Italy

Scott Dee

USA

Odir Dellagostin

Brazil

Mauro Delogu

Italy

Sagi Denenberg

Canada

Matthew Denwood

UK

Klaus Depner

Germany

Veronique Dermauw

Belgium

Robert Desrosiers

Canada

Johann Detilleux

Belgium

Andres Diaz

USA

Fco. Javier Diéguez

Spain

Chan Ding

China

Linda Dixon

UK

Cinzia Domeneghini

Italy

Mariano Domingo

Spain

Monique Dore

Canada

Jos Dortmans

Netherlands

Sara Downs

UK

Barbara Drews

Switzerland

Bert Driessen

Belgium

Tod Drost

USA
Ana Duarte

Portugal

Caroline Dube

Canada

Damien Dubois

France

Edward Dubovi

USA

Jennifer Duncan

UK

Stephen Dunham

UK

Magdalena Dunowska

New Zealand

Céline Dupuy

France

Michael Dykstra

USA

Sue Dyson

UK

Francis Dziva

Trinidad and Tobago

Peter David Eckersall

UK

Syed Ehtisham Ul Haque

Pakistan

Yvonne Eiby

Australia

Armin Elbers

Netherlands

Yvonne Elce

Canada

Steve Elder

USA

William Ellis

UK

Mansour El-Matbouli

Austria

Ingegerd Elvers

USA

Carsten Enevoldsen

Denmark

Francis Enjalbert

France 
Luis Enjuanes

Spain

Kira Epstein

USA

Ronald Erskine

USA

Mark Erwin

Canada

Matthieu Eveillard

France

Darrick Evensen

USA

Christelle Fablet

France

Maria Fahie

USA

Mohamed Fakhr

USA

Kiterie Faller

UK

Yunpeng Fan

China

Mark Farnworth

New Zealand

Vahab Farzan

Canada

Maren Fedrowitz

Germany

Daniel Feeney

USA

Li Feng

China

Aude Ferran

France

Edmilson Ferreira De Oliveira Filho Belgium

Carrie Finno

USA

Nahuel Fittipaldi

Canada

Paul Flecknell

UK

Krzysztof Flisikowski

Germany
Michael Flythe

USA

Steven Foley

USA

Anthony Fooks

UK

Ivo Foppa

USA

Hubert Forster

Jordan

Geoffrey Foster

UK

Gilles Foucras

France

Guillaume Fournié

UK

Leo Foyle

Australia

Carlos Franco

Spain

Dimitrios Frangoulidis

Germany

Conrad Freuling

Germany

Schirin Friederichs

Germany

Anika Friese

Germany

Jean-Pierre Frossard

UK

George Fthenakis

Greece

Clement Furlong

USA

Malgorzata Gajewska

Poland

Vladimir Galindo-Zamora

Colombia

Marina Gallo Calderon

Argentina

Patricia Gama

Brazil

Gualtiero Gandini

Italy
Ana L. Garcia-Perez

Spain

Mutien-Marie Garigliany

Belgium

David Gasper

USA

Charles Gauci

Australia

Anne Geffré

France

Claudio Genchi

Italy

Solange Gennari

Brazil

Arcangelo Gentile

Italy

Ivaylo Gentschev

USA

Raymond Geor

USA

Kerstin Gerlach

Germany

Pierre Germon

France

Andrea Giacometti

Italy

Ilias Giannenas

Greece

Mirutse Giday

Ethiopia

Pedro J. Ginel

Spain

Mario Giorgi

Italy

Etienne Giraud

France

Liz Glass

UK

Mindy Glass

USA

Helen Golder

Australia

Rita Goncalves

UK 


\author{
Lorenzo Gonzalez \\ UK \\ David González-Barrio \\ Spain \\ Daniela Gorgas \\ Switzerland \\ Marga Goris \\ Netherlands \\ Eamonn Gormley \\ Ireland \\ Simon Paul Graham
UK \\ Simon Graham \\ UK \\ Iain Grant \\ UK \\ Kathryn Graves \\ USA \\ Gilbert Greub \\ Switzerland \\ Deborah Grosenbaugh \\ USA \\ Sigrid Grulke \\ Belgium
}

Alonso Guedes

USA

Roberto Guedes

Brazil

Bruce Gummow

Australia

Sanjeev Gupta

Ireland

Franco Guscetti

Switzerland

Carlton Gyles

Canada

Adriana Györke

Romania

Bernd Haas

Germany

Daniela Christina Hadorn

Switzerland

Olga Haenen

etherlands
Ferry Hagen

Netherlands

Mitika Hagiwara

Brazil

Micah Hahn

USA

Rodrigo Hamede

Australia

David Hampson

Australia

Jarrad Hampton-Marcell

USA

Sanni Hansen

Denmark

Tom Harcourt-Brown

UK

Timm Harder

Germany

Robert Harman

USA

Balazs Harrach

Hungary

Chris Harris

Canada

Kenton Hart

UK

Kirsten Hattermann

Germany

Eleanor Hawkins

USA

Qing He

USA

Patricia Hedenqvist

Sweden

Joanna Hedley

UK

Ibrahim Hegab

Korea, South

Nagendra Hegde

India

Mohammad Heidari USA

Markus M. Heimesaat

Germany
Wayne Hein

Australia

Marcos Heinemann

Brazil

Lucie Heinzerling

Germany

Chris Helps

UK

Joerg Henning

Australia

Christina Herold

Germany

Pedro Herraez

Spain

Georg Herrler

Germany

Wolfgang Heuwieser

Germany

Marion Hewicker-Trautwein

Germany

Janet Hill

Canada

Gen Hiyama

Japan

Douglas Hodgins

Canada

Jane Hodgkinson

UK

Chris Hodgson

UK

Doris Hoeltig

Germany

Detlef Hoereth-Boentgen

Germany

Martin Hofmann

Switzerland

Erik Hofmeister

USA

Astrid Hogenkamp

Netherlands

Odd Viking Höglund

Sweden

Ole Hojberg

Denmark 
UK

Shannon Holmes

USA

Walther Honscha

Germany

Jayne Hope

UK

Robert Horton

UK

Margaret Hosie

UK

Herve Hoste

France

Xiuguo Hua

China

Bing Huang

China

Velia Hülsmeyer

Germany

Nora Hunter

UK

Jamal Hussaini

Malaysia

Changbaig Hyun

Korea, South

Eleonora Iacono

Italy

Yamamoto Ichiro

Japan

Jean-Claude Ionita

Germany

Re Isaacson

USA

Gloria Isani

Italy

Ramiro Isaza

USA

Naoki Isobe

Japan

Daisuke Ito

USA

Abdul Jabbar

Australia
Katarzyna Jagusztyn-Krynicka

Poland

George Jallo

USA

Henrik Elvang Jensen

Denmark

Trine Jensen

Denmark

Marianne Jensen-Waern

Sweden

Narayanaperumal Jeyathilakan

India

Jieyuan Jiang

China

Yonghou Jiang

China

Amy Johnson

USA

Christopher Johnson

USA

Timothy Johnson

USA

Clinton Jones

USA

Richard Jones

UK

Ronald Jones

UK

Chaitanya Joshi

India

Jennifer Lee Juengel

New Zealand

Jana Juránková

Czech Republic

Ramon A. Juste

Spain

Jaroslaw Kaba

Poland

Annemarie Kaesbohrer

Germany

Donata Kalthoff

Germany

Joshua Kamani

Nigeria
Ji-Houn Kang

Korea, South

Heike Kaspar

Germany

Anil Kumar Kataria

India

Frank Katzer

UK

Kevin Keegan

USA

Rob Kelly

UK

Michael Kent

USA

Mahesh Khatri

USA

Kamel Khazal

USA

Timothy Kidd

Australia

Manfred Kietzmann

Germany

Amy Kilbride

UK

Hyeun Bum Kim

Korea, South

Ralph Kirby

Taiwan

Carsten Kirkeby

Denmark

Jolle Kirpensteijn

Netherlands

William Kisseberth

USA

Jerzy Kita

Poland

Anna-Mariam Kiviranta

Finland

Wolfgang Klee

Germany

Eyal Klement

Israel

Robert Klopfleisch

Germany 
Derek Knottenbelt

UK

Guus Koch

Netherlands

Achim Kohler

Norway

Nicholas Komar

USA

Ganesh Kondabattula

India

Timm Konold

UK

Peter Kook

Switzerland

Hendrik-Jan Kranenburg

Netherlands

Peter Krawczel

USA

Magdalena Krol

Poland

Christa Kuehn

Germany

Hanna Kulig

Poland

Sachin Kumar

India

Nina Kung

Australia

Jacqueline Kupper

Switzerland

Nitin Kurkure

India

Ohkyeong Kweon

Korea, South

Roberto La Ragione

UK

Raphael Labens

UK

Jean-Paul Lallès

France

Abraham Landa

Mexico

Wil J.M. Landman

Netherlands
Paul Langford

UK

Carlos Lanusse

Argentina

Sasha Lanyon

Australia

Susanna Lau

China

Marcia Laurenti

Brazil

Richard Laven

New Zealand

Michael Lawman

Finland

Patricia Lawman

USA

Yves Le Loir

France

John Lednicky

USA

Jun Heon Lee

Korea, South

Tosso Leeb

Switzerland

Peter Lees

UK

Chu-Zhao Lei

China

Tiziana Lembo

UK

Carlo Leonetti

Italy

Henry L'Eplattenier

UK

Michael Leschnik

Austria

Bruno Levecke

Belgium

Charles Ley

Sweden

Jacqueline Ley

Australia

Erguang Li

China
Meihang Li

Canada

Robert Li

USA

Qu Liandong

China

Ai-Ho Liao

Taiwan

Geneviève Libeau

France

Brandon Lillie

Canada

Hai Lin

China

David Lindsay

USA

Susan Little

Canada

Chao-Lin Liu

Taiwan

Huili Liu

China

Changming Liu

China

Wenjun Liu

China

Qinfang Liu

USA

Xiufan Liu

China

Caryl Lockhart

Italy

Hannes Lohi

Finland

Sam Long

Australia

Alessio Lorusso Italy

Marta Lourenco

Belgium

James Lowe

USA

Huaguang Lu

USA 
Yu Lu

China

George Lubas

Italy

Stephen Luby

USA

Cecilia Lucero Estrada

Argentina

Jennifer Luff

USA

Stelio Luna

Brazil

Heidi Sjetne Lund

Norway

Xiaohu Luo

China

Agnese Lupo

Switzerland

Andrea Luppi

Italy

Bertrand Lussier

Canada

Inna Lysnyansky

Israel

N James Maclachlan

USA

James Macleod

USA

Dominiek Maes

Belgium

Raul Mainar-Jaime

Spain

Francesca Mancianti

Italy

Christoph Mans

USA

Karen Mansfield

UK

Lucia Manso-Silvan

France

Shengyong Mao

China

Ricardo Marcos

Portugal
Cinzia Marianelli

Italy

Lieta Marinelli

Italy

Gabriele Marino

Italy

Marga Martin

Spain

Guy-Pierre Martineau

France

Fernando Martinez

Spain

Silvia Martinez-Subiela

Spain

Daniele Martins

Brazil

Gabriel Martins

Brazil

Gift Matope

Zimbabwe

Augusto Matos

Portugal

Olga Matos

Portugal

Jelle Matthijnssens

Belgium

Meghan May

USA

Christie Mayo

USA

Brad Mccall

Australia

Scott Mcclure

USA

Neil Ross Mcewan

UK

John Mcgarry

UK

Colin Mcinnes

UK

Mary-Louise Mclaws

Australia

Barbara Mcmahill

USA
Bret Mcnabb

USA

Steven Mcorist

UK

Graham Medley

UK

Joanne Meers

Australia

Björn Meij

Netherlands

Antonio Melendez Lazo

Spain

Adriana Méndez Bernal

Mexico

Michael Mendl

UK

Estelle Méroc

Belgium

Alfred Merritt

USA

Ignacio Mesa-Sánchez

Spain

Andrea Mess

Brazil

Francois Meurens

Canada

Martin Michaelis

UK

Andrei Daniel Mihalca

Romania

Djordje Miljkovic

Serbia

Patti Miller

USA

Suzanne Millman

USA

L Scott Mills

USA

Rowan Milner

USA

Jose Roberto Mineo

Brazil

Takuya Mizuno

Japan 


\begin{tabular}{|c|c|c|}
\hline Shusei Mizushima & Pamela Murison & Chiara Noli \\
\hline Japan & UK & Italy \\
\hline David Modrý & Serge Muyldermans & Sarah North \\
\hline Czech Republic & Belgium & UK \\
\hline Giovanni Mogicato & Hanspeter Naegeli & Erik Noschka \\
\hline France & Switzerland & Australia \\
\hline Fouad Mohammad & Vinny Naidoo & Christopher Ober \\
\hline Iraq & South Africa & USA \\
\hline Matlou Mokgotho & Shan Naidu & Peter Thomas Oberem \\
\hline South Africa & USA & South Africa \\
\hline Jan Mol & Bindu Nanduri & Victoria Anne Offord \\
\hline Netherlands & USA & UK \\
\hline Fabiano Montiani-Ferreira & Richard Nap & Hiroyuki Okada \\
\hline Brazil & Argentina & Japan \\
\hline J. Alberto Montoya-Alonso & Sebastian Napp & Shoshiro Okada \\
\hline Spain & Spain & Japan \\
\hline Simon More & Heiko Nathues & Yuki Okada \\
\hline Ireland & Switzerland & Japan \\
\hline Maxim Moreau & Helena Neumayerova & Teresa Cristina Oliveira-Sequeira \\
\hline Canada & Czech Republic & Brazil \\
\hline Anabela Moreira & Mandy Nevel & Thierry Olivry \\
\hline Portugal & UK & USA \\
\hline Ana Moreno & Tzi Bun Ng & Pal A. Olsvik \\
\hline Italy & Hong Kong & Norway \\
\hline Juan Morgaz & Seongwon Nho & Oluyinka Olukosi \\
\hline Spain & Korea, South & UK \\
\hline Nobuko Mori & Robin Nicholas & Maarten Oosterlinck \\
\hline Japan & UK & Belgium \\
\hline Luca Motta & Tracy Nicholson & Tanja Opriessnig \\
\hline UK & USA & UK \\
\hline Vladimir Mrljak & Artur Niedzwiedz & Geert Opsomer \\
\hline Croatia & Poland & Belgium \\
\hline Gideon D Mshelia & Søren Achim Nielsen & Erman Or \\
\hline Nigeria & Denmark & Turkey \\
\hline Martha Mulks & Jarkko Niemi & Angel Ortiz-Pelaez \\
\hline USA & Finland & UK \\
\hline Thomas Muller & Stijn Niessen & Melania Osto \\
\hline Germany & UK & Switzerland \\
\hline Joachim Müller & Jorge Nieto & Tarja Pääkkönen (Previously Jokinen) \\
\hline Switzerland & USA & Finland \\
\hline Lina Mur & Akbar Nikkhah & José Ricardo Pachaly \\
\hline Spain & Iran & Brazil \\
\hline Atsushi Murai & Flobert Njiokou & Akos Pakozdy \\
\hline Japan & Cameroon & Austria \\
\hline
\end{tabular}


Saverio Paltrinieri

Italy

Lisa Pang

UK

Fernando Paolicchi

Argentina

Elias Papadopoulos

Greece

Kostas Papasouliotis

UK

Roberto Papini

Italy

Bart Pardon

Belgium

Elizabeth Parker

Australia

Tim Parkin

UK

Thomas Parsons

USA

Peter Pascoe

USA

Santiago Pascual

Spain

Urszula Paslawska

Poland

Josep Pastor

Spain

Gavin Paterson

UK

Nina Patzke

South Africa

Heather Paxton

UK

Rita Payan Carreira

Portugal

Laura Peachey

UK

David Pearl

Canada

Dannele Peck

USA

Christian Peham

Austria
Xin-Hai Pei

USA

Simone Peletto

Italy

Li Peng

China

Bruno Penna

Brazil

Louis Penning

Netherlands

Mary Louise Penrith

South Africa

Felipe Perecin

Brazil

Sandro Pereira

Brazil

Andres Perez

USA

Dolores Perez Alenza

Spain

Bernat Pérez De Val

Spain

Jose Perez-Casal

Canada

Michael Peterson

USA

Rob Pettitt

UK

Thilo Pfau

UK

Dirk Pfeiffer

UK

Adrian Philbey

UK

Giuseppe Piccione

Italy

Alessandra Piccirillo

Italy

Kim Picozzi

UK

Tina Pihl

Denmark

Gina Pinchbeck

UK
Eric Pinloche

UK

Patrick Pithua

USA

Alessandro Poli

Italy

Iuliana Popa

France

Thibaud Porphyre

UK

Heidrun Potschka

Germany

Andrew Potts

South Africa

Barbara Powers

USA

Barbara Pratscher

Austria

Katy Proudfoot

USA

Chris Proudman

UK

Anna Puigdemont

Spain

Felisbina Queiroga

Portugal

Rupert Quinnell

UK

Wagner Quintilio

Brazil

Chantal Ragetly

France

Sheila Canevese Rahal

Brazil

Zia Rahman

Pakistan

Pascal Rainard

France

Ana S. Ramirez

Spain

Alejandro Ramirez

USA

Vijay Ramiya

USA 
Thaha Rasool

United Arab Emirates

Nicki Reed

UK

Phil Reeves

Australia

Ralf Regenthal

Germany

Javier Regidor-Cerrillo

Spain

Habib Rehman

Pakistan

Michael Reichel

Australia

Richard Reid-Smith

Canada

Janin Reifenrath

Germany

Kerstin Reimers

Germany

Craig Reinemeyer

USA

William Reisen

USA

Monika Reissmann

Germany

Alexander Reiter

USA

Anne Relun

France

David Renaudeau

Finland

Subramaniam Renuka

USA

Crawford Revie

Canada

Brice Reynolds

France

Federica Riccardo

Italy

Rose Eli Rici

Brazil

Christopher Riley

New Zealand
Gerald Rimbach

Germany

Laura Rinaldi

Italy

Robert Ringseis

Germany

Cecilia Robat

USA

Helen Roberts

UK

Mara Rocchi

UK

Dominique Rocha

France

Annie Rodolakis

France

Fernando Rodriguez

Spain

Carlos Rodriguez

USA

Simon Roe

USA

Bernard Roelen

Netherlands

Cecilia Rohdin

Sweden

Angela Römer-Oberdörfer

Germany

Paola Roncada

Italy

Ruben Rosales

UK

Ricardo Rosenbusch

USA

Alexander William Ross

UK

Elizabeth Ross

Australia

Tanya Rossi

Canada

John Rossmeisl

USA

Dennis Rubbenstroth

Germany
Till Ruemenapf

Austria

Thomas Rutkowski

USA

Andrew Rycroft

UK

Marie-Pierre Ryser-Degiorgis

Switzerland

Mikel Sabater

Spain

Claude Sabeta

South Africa

Farhad Safarpoor Dehkordi

Iran

Musa Sahin

Turkey

Orhan Sahin

USA

Michelle Sait

Australia

Lazaros Sakkas

Greece

Yoshihiro Sakoda

Japan

Fidel San Román

Spain

Rodrigo Sanabria

Argentina

Javier Sanchez

Canada

Arturo Sanchez-Paz

Mexico

Berta São Braz

Portugal

Rafal Sapierzynski

Poland

Manolis Saridomichelakis

Greece

Tomohiro Sasanami

Japan

Jimmy Saunders

Belgium

Ioannis Savvas

Greece 
Claire Scantlebury

UK

Eugenio Scanziani

Italy

Sabine Schäfer-Somi

Austria

Kathrin Schemann

Australia

Julie Schmied

Canada

Lauren Schnabel

USA

Stephan Schumacher

Germany

Gerald Fritz Schusser

Germany

Stefan Schwarz

Germany

Heinzpeter Schwermer

Switzerland

Ignacio Segarra

Spain

Alessandro Seguino

UK

Hermann Seifert

Germany

Tsutomu Sekizaki

Japan

Thomas Selhorst

Germany

Kim Selting

USA

Martin Sergeant

UK

Torsten Seuberlich

Switzerland

Hans-Martin Seyfert

Germany

Nesrin Seyhan

Turkey

Tongling Shan

China

Sudarvili Shanthalingam

USA
Alexandra Shaw

UK

Wanyu Shi

China

Workineh Shibeshi

Ethiopia

Barbara Shock

USA

Meng Siak

Australia

Marina Sibila

Spain

Alexandre Rodrigues Silva

Brazil

Pedro Silva

Brazil

Rodrigo Otávio Silva

Brazil

Hubert Simhofer

Austria

Nagendrakumar Singanallur

Australia

Mini Singh

Australia

Raj Kumar Singh

India

Pavel Siroky

Czech Republic

Patchima Sithisarn

Thailand

Katherine Skorupski

USA

Philip Skuce

UK

Jan Slapeta

Australia

Josh Slater

UK

Ronald Sluyter

Australia

Hilde Smith

Netherlands

Richard Smith

UK
Laura Soler Vasco

Belgium

Anongnart Somchit-Assavacheep

Thailand

Kun-Ho Song

Korea, South

Victoria Soroker

UK

Eva Spada

Italy

Jessica Sparkes

Australia

Monica Sparo

Argentina

Joachim Spergser

Austria

Subramaniam Srikumaran

USA

Judith Stack

UK

Francesco Staffieri

Italy

Jessica Stahl

Germany

Karl Stahl

Sweden

Catherine Stalin

UK

Amy L Stanton

USA

Christoph Staubach

Germany

Jenny Stavisky

UK

Frik Stegmann

South Africa

Timothy Stein

USA

Sarah Steinbach

Germany

Hs Steinberg

USA

Jevrosima Stevanovic

Serbia 


\begin{tabular}{|c|c|c|}
\hline Kim Stevens & Florence Tardy & Imke Traulsen \\
\hline UK & France & Germany \\
\hline Mark Stevenson & Mike Taylor & Paolo Trevisi \\
\hline Australia & UK & Italy \\
\hline Mark Stevenson & Marta Teixeira & Annabelle Troegeler \\
\hline New Zealand & Brazil & France \\
\hline George Stilwell & Marisa Tellez & Troy Trumble \\
\hline Portugal & USA & USA \\
\hline Reinhard Sting & Doga Temizsoylu & Marianna Tryfonidou \\
\hline Germany & Turkey & Netherlands \\
\hline Norbert Stockhofe-Zurwieden & Calogero Terregino & Panagiotis Tsikouras \\
\hline Netherlands & Italy & Greece \\
\hline Diana Stone & Eileen Thacker & Romana Turk \\
\hline Grenada & USA & Croatia \\
\hline Viola Strompfová & Douglas Thamm & Stanislawa Tylewska-Wierzbanowska \\
\hline Slovakia & USA & Poland \\
\hline Rebecca Strong & Alexandre Thibodeau & Jouni Uitto \\
\hline UK & Canada & USA \\
\hline Shuo Su & Charles Thoen & Reiner Ulrich \\
\hline China & USA & Germany \\
\hline Hiromu Sugiyama & Peter Thompson & Hermann Unger \\
\hline Japan & South Africa & Austria \\
\hline Jianhe Sun & Peter T. Thomsen & Melissa Upjohn \\
\hline China & Denmark & UK \\
\hline Lakshmi Sunkara & Gavin Thomson & Yves Van Der Stede \\
\hline USA & Zambia & Belgium \\
\hline Gila Sutton & Barbara Thur & Leo Van Leengoed \\
\hline Israel & Switzerland & Netherlands \\
\hline Pavol Svorc & Deena Tiches & Hugo Van Oostrom \\
\hline Slovakia & USA & Netherlands \\
\hline Emmanuel Swai & Andrea Tipold & Paul René Van Weeren \\
\hline Tanzania & Germany & Netherlands \\
\hline Kelly Swanson & Laurent Tiret & Katrien Vanderperren \\
\hline USA & France & Belgium \\
\hline Jane Sykes & Mike Tokach & Raphael Vanderstichel \\
\hline USA & USA & Canada \\
\hline Monika Szymanska-Czerwinska & Montserrat Torremorell & Marc Vandevelde \\
\hline Poland & USA & Switzerland \\
\hline Sabine Tacke & Sabine Totemeyer & Jean-Michel Vandeweerd \\
\hline Germany & UK & Belgium \\
\hline John S. Tam & Philippe Totte & An Vanhaesebrouck \\
\hline Hong Kong & France & UK \\
\hline Qinghai Tang & Pierre-Louis Toutain & Mary Varaschin \\
\hline China & France & Brazil \\
\hline
\end{tabular}




\begin{tabular}{|c|c|c|}
\hline $\begin{array}{l}\text { Luigi Varesio } \\
\text { Italy }\end{array}$ & $\begin{array}{l}\text { Hui Wang } \\
\text { USA }\end{array}$ & $\begin{array}{l}\text { Benjamin Willing } \\
\text { Canada }\end{array}$ \\
\hline $\begin{array}{l}\text { Celina Vega } \\
\text { Argentina }\end{array}$ & $\begin{array}{l}\text { Jiaqi Wang } \\
\text { China }\end{array}$ & $\begin{array}{l}\text { Sandra Wilsher } \\
\text { UK }\end{array}$ \\
\hline $\begin{array}{l}\text { Monica Venner } \\
\text { Germany }\end{array}$ & $\begin{array}{l}\text { Qin Wang } \\
\text { China }\end{array}$ & $\begin{array}{l}\text { Stephen Wilson } \\
\text { Belgium }\end{array}$ \\
\hline $\begin{array}{l}\text { Claudio Vergari } \\
\text { France }\end{array}$ & $\begin{array}{l}\text { Scott Weese } \\
\text { Canada }\end{array}$ & $\begin{array}{l}\text { Angela Witzel } \\
\text { USA }\end{array}$ \\
\hline $\begin{array}{l}\text { Tiziano Verri } \\
\text { Italy }\end{array}$ & $\begin{array}{l}\text { Patrick Wefstaedt } \\
\text { Germany }\end{array}$ & $\begin{array}{l}\text { R. Mark Wooten } \\
\text { USA }\end{array}$ \\
\hline $\begin{array}{l}\text { Haralabos Ververidis } \\
\text { Greece }\end{array}$ & $\begin{array}{l}\text { Qiang Wei } \\
\text { China }\end{array}$ & $\begin{array}{l}\text { Deanna Worley } \\
\text { USA }\end{array}$ \\
\hline $\begin{array}{l}\text { Flavie Vial } \\
\text { Switzerland }\end{array}$ & $\begin{array}{l}\text { Marleen Werkman } \\
\text { UK }\end{array}$ & $\begin{array}{l}\text { Lihua Xiao } \\
\text { USA }\end{array}$ \\
\hline $\begin{array}{l}\text { Joaquin Vicente } \\
\text { Spain }\end{array}$ & $\begin{array}{l}\text { Kerstin Wernike } \\
\text { Germany }\end{array}$ & $\begin{array}{l}\text { Ting Xu } \\
\text { China }\end{array}$ \\
\hline $\begin{array}{l}\text { Enric Vidal } \\
\text { Spain }\end{array}$ & $\begin{array}{l}\text { Björn Weström } \\
\text { Sweden }\end{array}$ & $\begin{array}{l}\text { Seiji Yamamoto } \\
\text { Japan }\end{array}$ \\
\hline $\begin{array}{l}\text { Carlos Viegas } \\
\text { Portugal }\end{array}$ & $\begin{array}{l}\text { Ulrik Westrup } \\
\text { Denmark }\end{array}$ & $\begin{array}{l}\text { Hanchun Yang } \\
\text { China }\end{array}$ \\
\hline $\begin{array}{l}\text { Jose Manuel Vilar } \\
\text { Spain }\end{array}$ & $\begin{array}{l}\text { Nick Wheelhouse } \\
\text { UK }\end{array}$ & $\begin{array}{l}\text { Qian Yang } \\
\text { China }\end{array}$ \\
\hline $\begin{array}{l}\text { Amy Vincent } \\
\text { USA }\end{array}$ & $\begin{array}{l}\text { Paul Wichgers Schreur } \\
\text { Netherlands }\end{array}$ & $\begin{array}{l}\text { Yuanchao Ye } \\
\text { USA }\end{array}$ \\
\hline $\begin{array}{l}\text { Charles Vite } \\
\text { USA }\end{array}$ & $\begin{array}{l}\text { Barbara Wieland } \\
\text { Mongolia }\end{array}$ & $\begin{array}{l}\text { Jung-Yong Yeh } \\
\text { Korea, South }\end{array}$ \\
\hline $\begin{array}{l}\text { Anastasia Vlasova } \\
\text { USA }\end{array}$ & $\begin{array}{l}\text { Paul Wigley } \\
\text { UK }\end{array}$ & $\begin{array}{l}\text { Kadir Yesilbag } \\
\text { Turks and Caicos Islands }\end{array}$ \\
\hline $\begin{array}{l}\text { Andrea Vögtlin } \\
\text { Switzerland }\end{array}$ & $\begin{array}{l}\text { Rebecca Wilkes } \\
\text { USA }\end{array}$ & $\begin{array}{l}\text { Zeki Yilmaz } \\
\text { Turkey }\end{array}$ \\
\hline $\begin{array}{l}\text { Brigitte Von Rechenberg } \\
\text { Switzerland }\end{array}$ & $\begin{array}{l}\text { Colin Wilks } \\
\text { Australia }\end{array}$ & $\begin{array}{l}\text { Xin Yin } \\
\text { China }\end{array}$ \\
\hline $\begin{array}{l}\text { Zoran Vrbanac } \\
\text { Croatia }\end{array}$ & $\begin{array}{l}\text { Lucas Willems } \\
\text { Belgium }\end{array}$ & $\begin{array}{l}\text { Han Sang Yoo } \\
\text { Korea, South }\end{array}$ \\
\hline $\begin{array}{l}\text { Joe Wakshlag } \\
\text { USA }\end{array}$ & $\begin{array}{l}\text { Brian Willett } \\
\text { UK }\end{array}$ & $\begin{array}{l}\text { Yukinori Yoshimura } \\
\text { Japan }\end{array}$ \\
\hline $\begin{array}{l}\text { Cheryl Waldner } \\
\text { Canada }\end{array}$ & $\begin{array}{l}\text { Andrew Williams } \\
\text { Denmark }\end{array}$ & $\begin{array}{l}\text { Megan Young } \\
\text { Australia }\end{array}$ \\
\hline $\begin{array}{l}\text { Per Wallgren } \\
\text { Sweden }\end{array}$ & $\begin{array}{l}\text { David Williams } \\
\text { Uganda }\end{array}$ & $\begin{array}{l}\text { Muhammad Shahbaz Yousaf } \\
\text { Pakistan }\end{array}$ \\
\hline $\begin{array}{l}\text { John Walmsley } \\
\text { UK }\end{array}$ & $\begin{array}{l}\text { Tim Williams } \\
\text { UK }\end{array}$ & $\begin{array}{l}\text { Zhongtang Yu } \\
\text { USA }\end{array}$ \\
\hline $\begin{array}{l}\text { Michael Wang } \\
\text { USA }\end{array}$ & $\begin{array}{l}\text { Diana Williams } \\
\text { UK }\end{array}$ & $\begin{array}{l}\text { Gui-Hong Zhang } \\
\text { China }\end{array}$ \\
\hline
\end{tabular}


Jin Zhang

USA

Jianqiang Zhang

USA

Longxian Zhang

China

Shuning Zhang

USA

Feng-Qi Zhao

USA
Junlong Zhao

China

Bin Zhou

China

En-Min Zhou

China

Xing-Quan Zhu

China

Alessandro Zotti

Italy
Feng-Cai Zou

China

Elizabeth Zumbrun

USA

Gila Zur

Israel 